This item was submitted to Loughborough's Research Repository by the author.

Items in Figshare are protected by copyright, with all rights reserved, unless otherwise indicated.

\title{
Performance evaluation of a digital electrical impedance tomography system
}

PLEASE CITE THE PUBLISHED VERSION

http://www.actapress.com/

\section{PUBLISHER}

(c) Acta Press

\section{VERSION}

AM (Accepted Manuscript)

\section{LICENCE}

CC BY-NC-ND 4.0

\section{REPOSITORY RECORD}

Ayati, S. Bentolhoda, Kaddour Bouazza-Marouf, David Kerr, and Michael D. O'Toole. 2019. "Performance Evaluation of a Digital Electrical Impedance Tomography System". figshare. https://hdl.handle.net/2134/9947. 
This item was submitted to Loughborough's Institutional Repository (https://dspace.lboro.ac.uk/) by the author and is made available under the following Creative Commons Licence conditions.

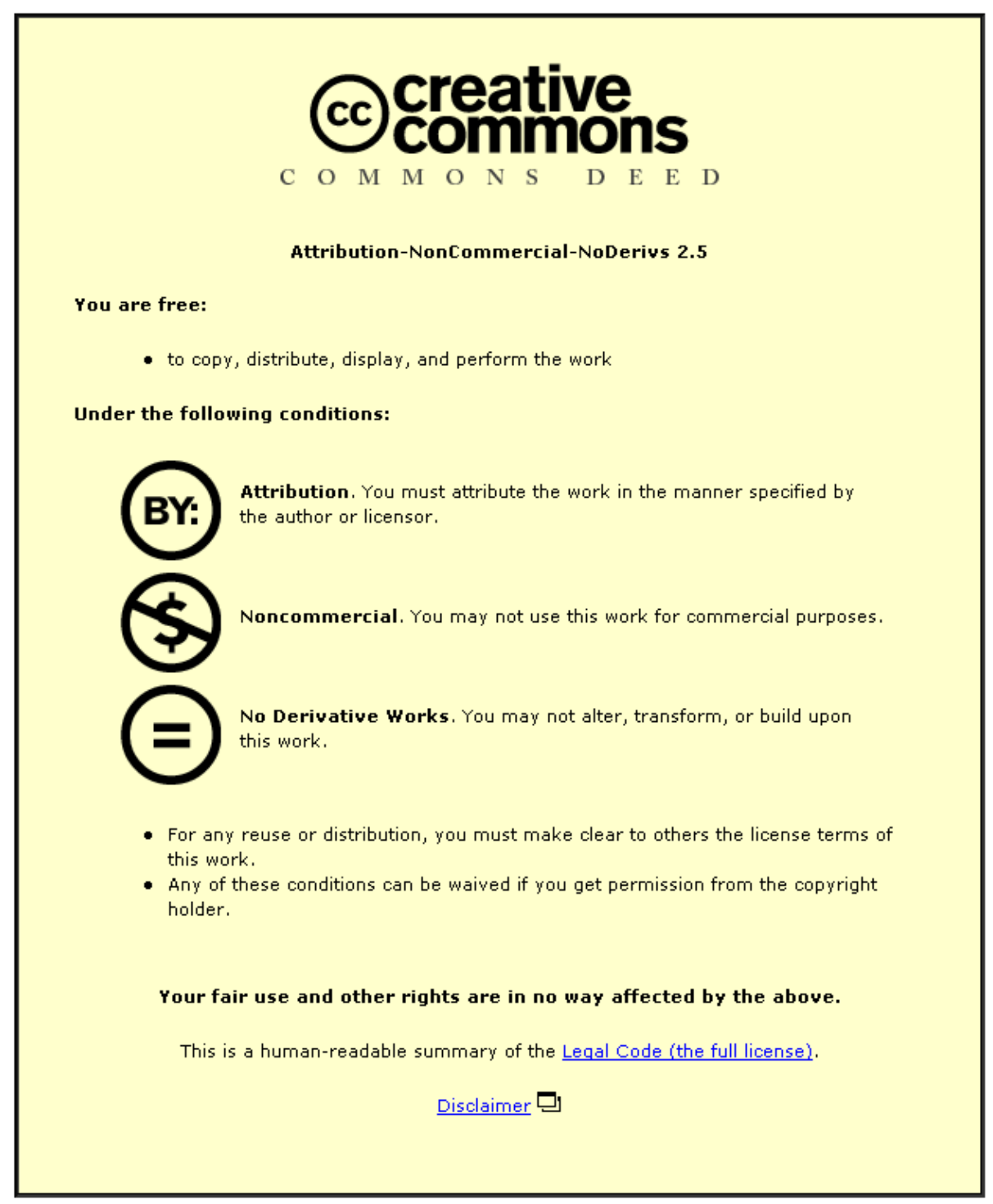

For the full text of this licence, please go to: http://creativecommons.org/licenses/by-nc-nd/2.5/ 


\title{
PERFORMANCE EVALUATION OF A DIGITAL ELECTRICAL IMPEDANCE TOMOGRAPHY SYSTEM
}

\author{
S. Bentolhoda Ayati ${ }^{\mathrm{a}}$, Kaddour Bouazza-Marouf ${ }^{\mathrm{b}}$, David Kerr ${ }^{\mathrm{c}}$, Michael O’Toole ${ }^{\mathrm{d}}$ \\ a, b, c Wolfson School of Mechanical and Manufacturing Engineering, Loughborough University, Loughborough, LE11 3TU, \\ UK \\ ${ }^{\mathrm{d}}$ School of Computer Science, University of Manchester, Oxford Rd, Manchester, M13 9PL, UK \\ aas.b.ayati@lboro.ac.uk, ${ }^{\mathrm{b}}$ K.Bouazza-marouf@lboro.ac.uk, ${ }^{\mathrm{c} D}$.Kerr@lboro.ac.uk, ${ }^{\mathrm{d}}$ Michael.OToole@cs.man.ac.uk
}

\begin{abstract}
Performance evaluation of a portable digital multifrequency electrical impedance tomography system is presented. The instrumentation hardware and image reconstruction are assessed according to a systematic methodology using a practical phantom. The phantom is equipped with eight electrodes in a ring configuration and a sinusoidal current of constant amplitude is injected using an adjacent current injection protocol. Artificial anomalies are introduced as inhomogeneity targets and the boundary potential data is collected. The images are reconstructed from the boundary data using Comsol Multiphysics and Matlab. Signal to noise ratio (SNR) and accuracy of the measurements are calculated. The limits of detectability and distinguishability of contrasts are measured from the collected potential data set for single and double inhomogeneities. The conductivity of the targets is successfully reconstructed from the potential data measurements. The detectability value is found to be high when a single target is close to the electrodes, while the values are less for the target in the centre. Also, the value of distinguishability increases when the targets move further away from each other.
\end{abstract}

\section{KEY WORDS}

Electrical Impedance Tomography System, phantom, Assistive Medical Technology, Medical Image Processing

\section{Introduction}

Internal hemorrhages are typically diagnosed using MRI or CT scan in hospitals; however, these imaging techniques are not portable and suitable for continuous monitoring and involve large personnel or equipment cost. Electrical impedance tomography (EIT) is a portable, noninvasive, non-radiating, non-ionizing and inexpensive technique which has the capability of long term monitoring to approximately localize the bleeding site. EIT is a medical imaging technique which reconstructs cross-sectional images of the electrical property distribution of an object within a body based on voltage measurements on its boundary. Data acquisition is performed through an array of electrodes which are attached to the surface of the body while small alternating electrical currents are injected into the electrodes [1]. EIT has been extensively researched in various clinical applications [2] due to its potential to distinguish between healthy and abnormal components of an organ based on the electrical property variation of the components according to their state of health [3]

Early EIT systems were designed using mainly analogue techniques with many associated problems such as noise, component mismatching, input offset, etc. The speed of previous analogue designs was also limited by factors such as switching transients, multiplexing overhead and conversion delays. Nowadays, EIT systems widely use digital techniques with the advantages of high stability and low noise. The most recent EIT systems are based on advanced technologies which improve their performance such as high bandwidth and good precision. Moreover, using digital components makes upgrading and debugging easier [4].

It is essential to evaluate and calibrate the performance of an EIT system as an assistive medical system before use, based on a systematic methodology using a practical phantom [5]. The goal of this paper is to evaluate the performance of a digital multi-frequency EIT system constructed in our laboratory as a prototype system for medical applications. The instrumentation hardware and image reconstruction are assessed. The tests are divided into a category of measurements of accuracy and a category of measurements of detectability.

\section{Materials and Methods}

\subsection{System Specification}

The structure of the designed EIT system based on a microcontroller is presented in Figure 1 . The system is divided into Microcontroller Unit (MCU), Direct Digital Synthesizer (DDS), Constant Current Source (CCS), Multiplexer and Demultiplexer (MUX/DEMUX) and Instrumentation Amplifier (IA) subsystems. The prototype model has 16 channels and operates in the frequency range of $25 \mathrm{kHz}$ to $100 \mathrm{kHz}$ with the temporal resolution of 100 frames per second. In order to control the data acquisition process, check electrode contact, visualize data and image, the system is connected to a PC through a serial port (RS232). System software, including graphical 


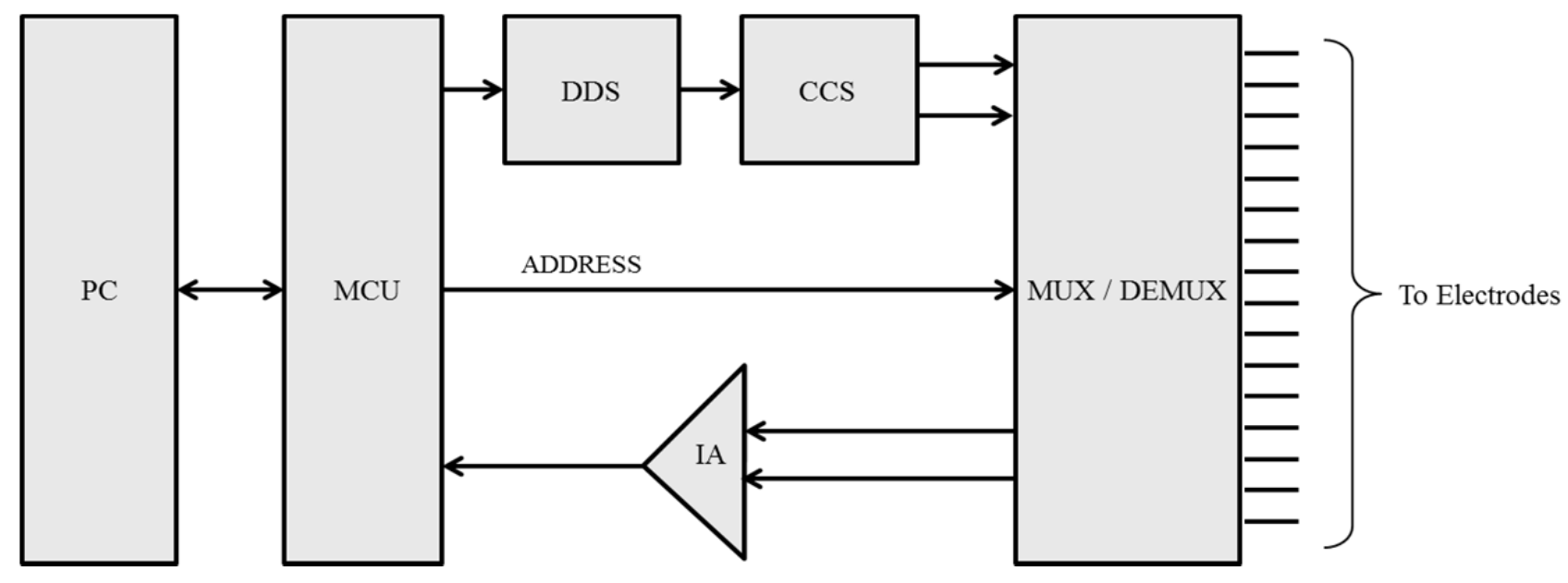

Figure 1. EIT system architecture

user interface, is developed using Visual Basic (VB).

\subsection{Phantom Configuration}

The EIT system is evaluated using phantom experiments in the presence of realistic noise. The phantom system consists of a shallow nylon tank, eight electrodes, the background solution, and the simulated anomalies (cucumber segments). The tank has a depth of $80 \mathrm{~mm}$ and an inner diameter of $144 \mathrm{~mm}$. The phantom is equipped with 8 stainless steel screws as point electrodes (dia. 10 $\mathrm{mm}$ ) equally spaced in a ring around the tank wall. All the lead wires are of equal lengths to obtain an identical impedance path through all the electrodes in order to reduce the mismatch in electrode impedances. $0.38 \%$ saline solution with the conductivity of approximately 1 $\mathrm{S} / \mathrm{m}$ is used for the background solution. Cylindrical pieces of cucumber with the conductivity of $0.05 \mathrm{~S} / \mathrm{m}$ are used as anomalies. The anomalies have a diameter of approximately $40 \mathrm{~mm}$ and the same height as the tank

\subsection{Data Collection and Image Reconstruction}

With reference to Figure 1, the CCS (NE5534, Texas Instruments) generates a constant sinusoidal current of $1 \mathrm{~mA}, 50 \mathrm{kHz}$ frequency. It is fed by a sinusoidal signal (1 $\mathrm{V}$ (rms)) of the same frequency which is generated by the DDS (AD9850, Analogue Devices). The sinusoidal current is injected into the phantom through one pair of adjacent electrodes and the corresponding boundary potentials are measured over pairs of the remainder of the neighbouring electrodes. The voltages are measured while the current is applied to the phantom. The input pair of electrodes is switched over all adjacent electrode pairs and the measurement procedure is repeated for all possible adjacent pairs to produce a complete voltage data set according to the adjacent electrode switching protocol. The measured differential potentials are amplified using the IA (PGA202KP, Texas Instruments). The amplified analogue signal is converted to digital and transferred to the PC using the MCU (PIC18F4620).
The images are reconstructed using Comsol Multiphysics and Matlab. The phantom domain is simulated as a 2D disk model with 8 electrodes. The domain is discretized to 1336 second-order triangular finite elements. Each pixel of the reconstructed images is related to the conductivity changes $\Delta \sigma$, which in turn are related to the differential potential $\Delta \mathrm{V}$, measured over the electrodes. This relationship in matrix form is expressed as a system of linear equations:

$$
\Delta \mathrm{V} \cong \mathrm{S} \Delta \sigma
$$

where $\mathrm{S}$ is known as the sensitivity matrix. The problem is to find conductivity changes for given measured potentials and calculated sensitivity matrix. The potential data is collected from the phantom experiments and the sensitivity matrix is calculated for a circular model of the phantom with uniform conductivity. The sensitivity matrix is always severely ill-conditioned and has large condition number. In this study, the sensitivity matrix has a full rank of 20 and the condition number of the order of $10^{17}$. The Truncated Singular Value Decomposition (TSVD) method is used to regularize the inversion of the sensitivity matrix. The truncation point $k$, needs to be chosen carefully, less than or equal to the rank of the matrix, as it would otherwise produce inaccurate images. The truncation point is chosen as 16 in this study.

\subsection{Evaluation Methodology}

The performance of our EIT system is evaluated according to Yasin et al using a systematic approach. In order to assess the noise and accuracy of the system, an acquisition of a sequence of at least 100 frames of data are collected from the homogenous saline-filled tank before anomalies are introduced. A signal to noise ratio (SNR) is calculated to estimate the precision of measurements. The SNR is defined as the ratio of signal amplitude to noise level for each of the measurement channels: 

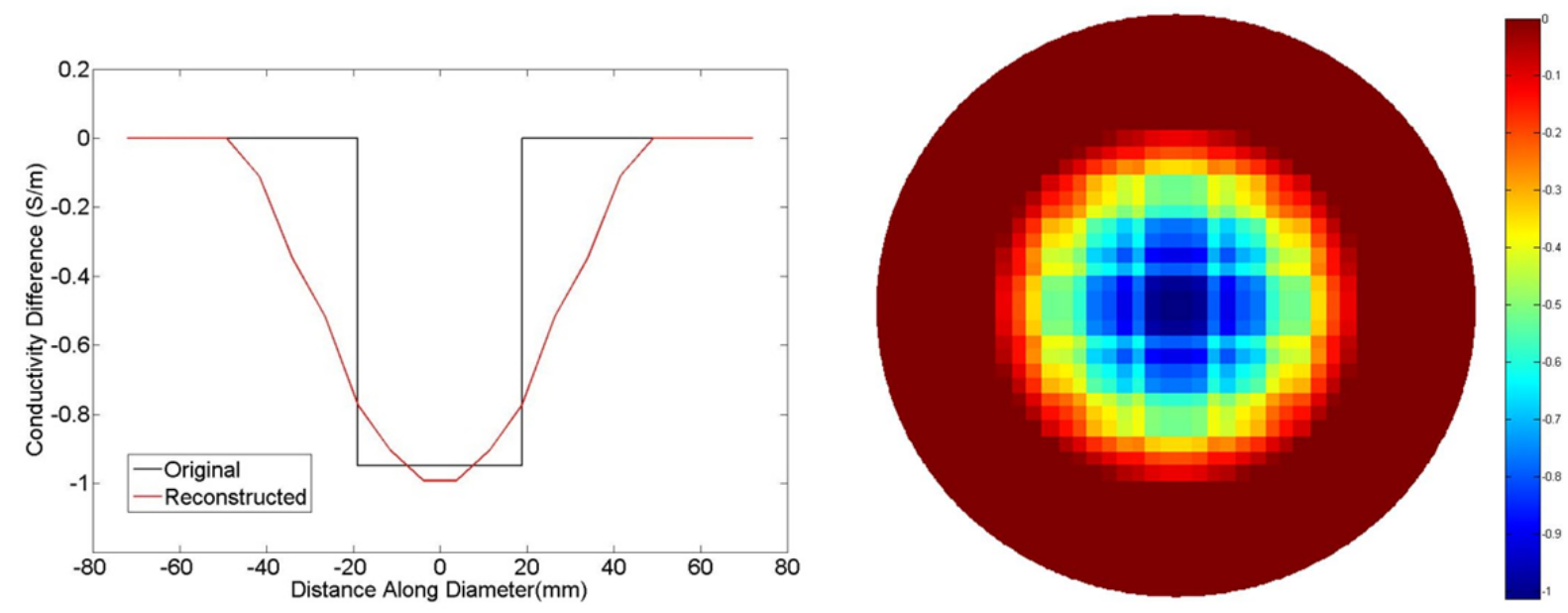

Figure 2. DRP and reconstructed image of phantom with one target at center
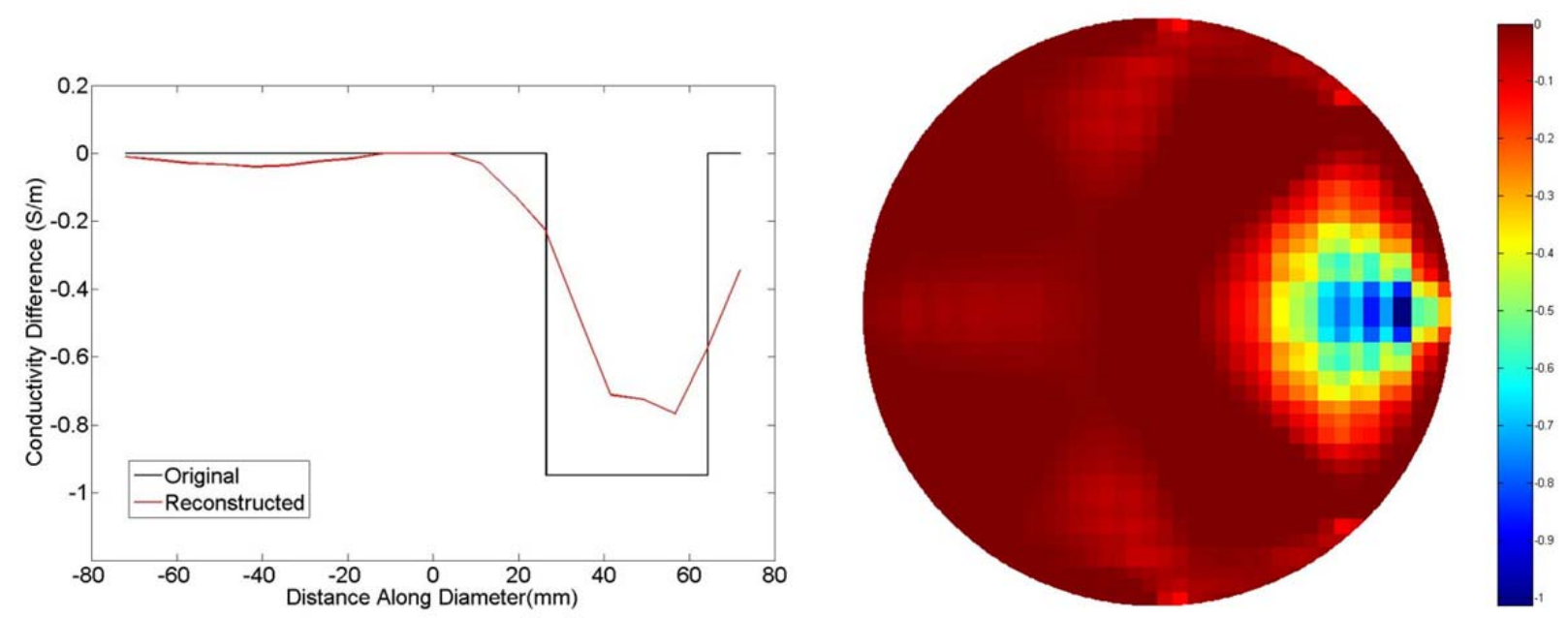

Figure 3. DRP and reconstructed image of phantom with one target close to edge of the tank at electrode No. 1
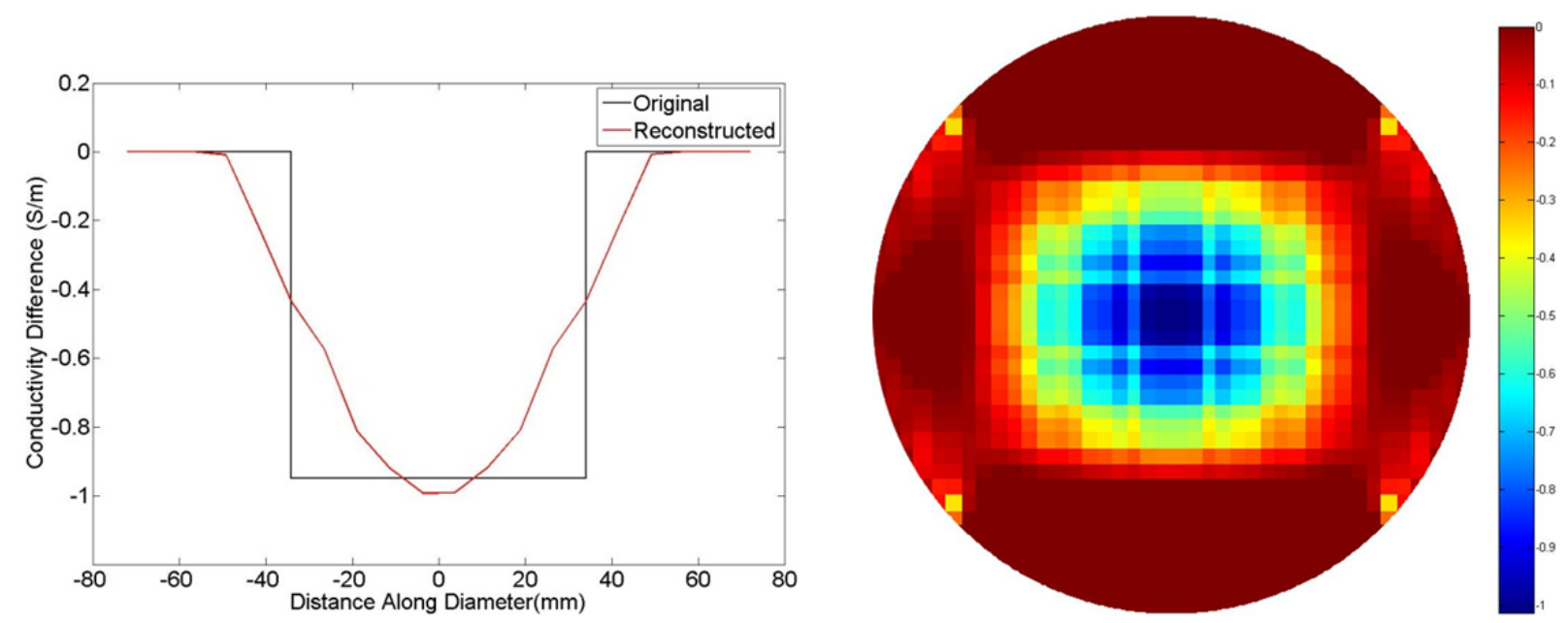

Figure 4. DRP and reconstructed image of phantom with two targets at center 

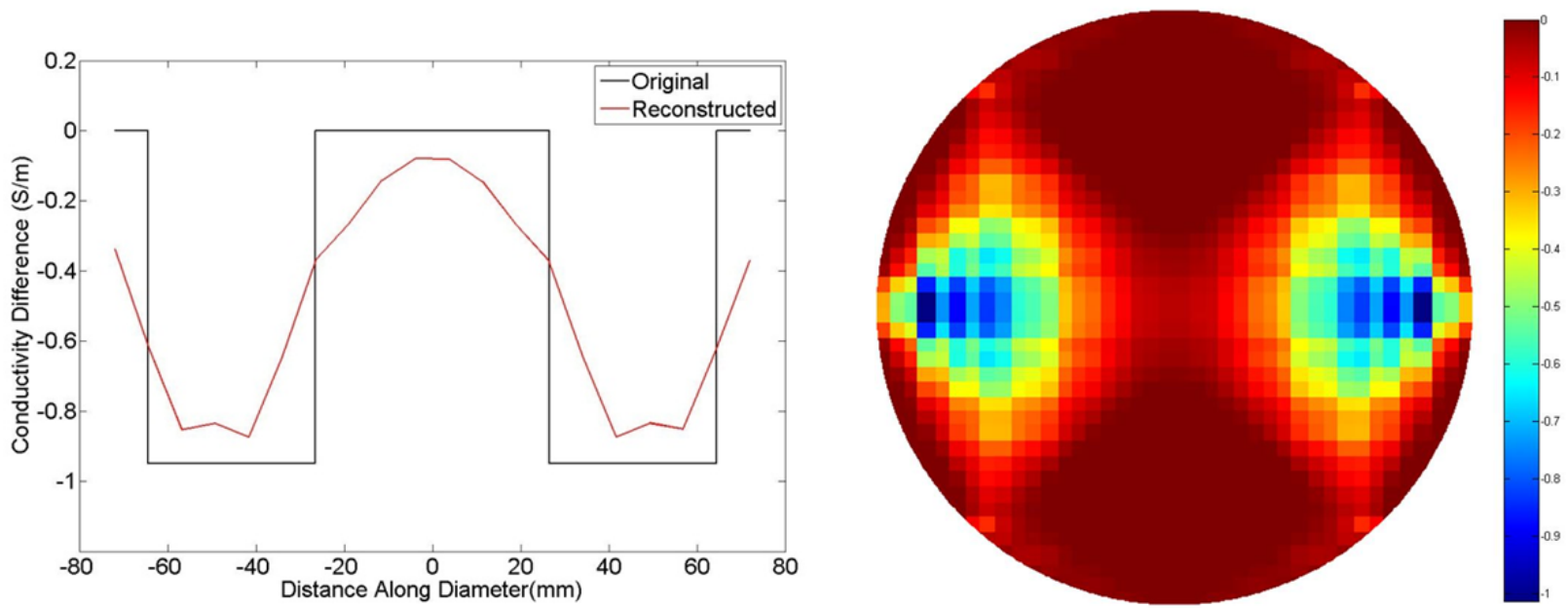

Figure 5. DRP and reconstructed image of phantom with two targets close to edge of the tank at electrodes No 1 and 5

$$
\mathrm{SNR}_{\mathrm{i}}=\frac{[\overline{\mathrm{V}}]_{\mathrm{i}}}{\mathrm{SD}_{[\mathrm{V}]_{\mathrm{i}}}}
$$

where $\mathrm{SD}_{[\mathrm{V}]_{\mathrm{i}}}$ is the standard deviation of multiple measurements for each channel and $[\overline{\mathrm{V}}]_{\mathrm{i}}$ is the mean value of the multiple measurements for each channel.

The closeness of the measured potential data, $\mathrm{V}$ to the estimated data set, $\mathrm{V}^{(\mathrm{sim})}$ generated by a Finite Element Model of the homogenous tank is described as Accuracy (AC) of a measurement system [6]. The simulated potentials are normalized to give a best fit to the real measurement obtained by phantom experiments. The accuracy of the $\mathrm{i}^{\text {th }}$ channel is

$$
\mathrm{AC}_{\mathrm{i}}=\left(1-\left|[\overline{\mathrm{V}}]_{\mathrm{i}}-\left[\mathrm{V}^{(\operatorname{sim})}\right]_{\mathrm{i}}\right|\right) \times 100 \%
$$

The limits of detectability and distinguishability of contrasts are measured after placing simulated inhomogeneities in the saline solution. The distinguishability is the ability to separate two adjacent targets from one while detectability is to distinguish the presence of a target from null (homogenous tank) [7]. Potential data are collected for single and multiple inhomogeneities placed at different locations in the phantom domain. Images are reconstructed from these measurements in order to determine the reliability of EIT detection of a single target, and of distinguishing two targets. $z$, given as,

$$
z=\frac{\widehat{\mathrm{X}}_{\mathrm{q}}}{s}
$$

is a measurement of the EIT system performance, where $\widehat{\mathrm{X}}_{\mathrm{q}}$ is the mean image in a region of interest (ROI), calculated from multiple reconstructed images and $s$ represents the standard deviation of multiple reconstructed

images. The ROI is selected and defined as image amplitude greater than $1 / 4$ of the maximum value rather than the entire image, in order to avoid contamination of the noise region by image artefacts.

DRP is the conductivity difference profile of the phantom model based on the phantom diameter passing through the reconstructed object center. It is plotted against the distance of the diameter [8].

\section{Results and Discussion}

Signal to noise ratio and accuracy are obtained from the measured data of the homogenous tank. The average SNR value of the channels is $43.1 \mathrm{~dB}$. The results show a high level of accuracy with an average accuracy of $93.5 \%$ for the system.

Figures 2 and 3 show DRP and the reconstructed images of the phantom with single target from the potential data. The images are successfully reconstructed and the targets at different positions are clearly detected. In the images the blue region which represents conductivity decreasing of $0.95 \mathrm{~S} / \mathrm{m}$ appeared in the image in accordance with the position of the target. The single target placed at the center is illustrated in Figure 2, and close to the edge of the tank at electrode No. 1 is shown in Figure 3. It is observed that the reconstructed DRPs almost follow the DRP of the original object.

DRP and the images of the phantom with two targets placed in the tank are illustrated in Figure 4 and 5. In the reconstructed images the main perturbation is produced close to the correct position with localized changes corresponding to the targets. Figure 4 shows the reconstruction of the two targets located at the center of the tank and very close to each other. The targets are detected but not distinguished clearly. In Figure 5 the targets are moved away from each other to the edge of the tank at electrodes No. 1 and 5. The targets are clearly 
detected and distinguished from each other and the reconstructed DRPs almost follow the DRP of the original object.

Detectability and distinguishability are calculated with respect to the position of the targets. The detectability values are found to be high, approximately 50, when a single target is close to the edge of the tank. However, when the target is at the centre of the tank, detectability reduced to around 20. The value of distinguishability increases from 25 to 55 as the targets move further away from each other.

\section{Conclusion}

The performance evaluation of the prototype model of the digital EIT system with a temporal resolution of 100 frames per second has been presented based on a systematic methodology using a practical phantom in the presence of realistic noise. The images of conductivity changes are successfully reconstructed with single and double inhomogeneities. The presented results demonstrate that our EIT system is sensitive to the conductivity variation and has the potential to produce reliable and localized images from the boundary potential measurements. These promising results of phantom experiments encourage further investigation on experiments using animal tissue for medical applications.

\section{References}

[1] J. G.Webster, Electrical impedance tomography (NY, Adam Hilger, 1990).

[2] D. S. Holder, Electrical impedance tomography: Methods, history and applications (GB, Institute of Physics, 2005).

[3] R. D. Stoy, K. R. Foster \& H. Schwan, Dielectric properties of mammalian tissues from 0.1 to $100 \mathrm{MHz}$; a summary of recent data, Physics in Medicine and Biology, 27, 1982, 501.

[4] M. R. Naeini, Low-noise measurement techniques for brain function imaging by electrical impedance tomography (University of Manchester, 2008).

[5] M. Yasin, S. Böhm, P. O. Gaggero \& A. Adler, Evaluation of EIT system performance, Physiological Measurement, 32(7), 2011, 851-865.

[6] H. Gagnon, M. Cousineau, A. Adler \& A. Hartinger, A resistive mesh phantom for assessing the performance of EIT systems, Biomedical Engineering, 57(9) 2010, 22572266.

[7] A. Adler, P. Gaggero \& Y. Maimaitijiang, Distinguishability in EIT using a hypothesis-testing model, Journal of Physics, Conference Series, 224, 2010.

[8] T. K. Bera and J. Nagaraju, Studying the resistivity imaging of chicken tissue phantoms with different current patterns in Electrical Impedance Tomography (EIT), Measurement, 45(4), 2012, 663-682. 